TITLE:

\title{
Multicountry modelling for the Japan Sea rim economic relations
}

$\operatorname{AUTHOR}(\mathrm{S})$ :

Ohnishi, Hiroshi; Yin, QingZhu

\section{CITATION:}

Ohnishi, Hiroshi ... [et al]. Multicountry modelling for the Japan Sea rim economic relations. 京都大学大学院経済学研究科Working Paper 2000, 49

\section{ISSUE DATE:}

2000

URL:

http://hdl.handle.net/2433/37932

RIGHT: 


\section{Multicountry Modelling for the Japan Sea Rim Economic Relations}

\section{Hiroshi Ohnishi}

Professor in the Graduate School of Economics, Kyoto University

\section{QingZhu Yin}

Graduate Student in the Graduate School of Economics, Kyoto University

Graduate School of Economics

Faculty of Economics

Kyoto University

Kyoto, 606-8501 JAPAN 


\section{Multicountry Modelling for the Japan Sea Rim Economic Relations}

\section{Hiroshi Ohnishi}

Professor in the Graduate School of Economics, Kyoto University

QingZhu Yin

Graduate Student in the Graduate School of Economics, Kyoto University 


\title{
Multicountry Modelling for the Japan Sea Rim Economic Relations
}

\author{
Hiroshi Ohnishi \\ Professor in the Graduate School of Economics, Kyoto University \\ QingZhu Yin \\ Graduate Student in the Graduate School of Economics, Kyoto University
}

\section{A survey of the Japan Sea Rim model}

Economic relations among the Japan Sea ${ }^{1}$ rim regions are going to be an increasingly important issue, particularly in the response to the Japan-Korea free trade area. Already a huge scale multicountry econometric model (NAMIOS I, see Shishido, et al (1999)) has been designed. It is maintained by ERINA (Economic Research Institute for Northeast Asia) ${ }^{2}$ and includes 7 countries (including USA), and can analyze international relations industry-by-industry. Further, it divides the Chinese sector into two; the Northeast region sector and another sector. Such a division is very important because the Northeast region is the nearest to and the most influential on the other Japan Sea rim countries. Moreover, the size of the sector's population (over 100 million) and its economic activity is thereby made comparable with those of other countries.

However, NAMIOS model's international trade bloc does not feature the Chinese Northeast sector. That is, the NAMIOS model includes the Chinese Northeast sector's macroeconomic model, but not its international trade bloc. Therefore, the NAMIOS model cannot directly analyze the trade relations with this region.

One purpose of the model presented in this paper is to overcome this weakness of the NAMIOS model. Using data in the China Statistical Yearbook and the three relevant provincial statistical yearbooks, we built a Chinese Northeast model that includes both the domestic macro model and the trade bloc. Therefore, this sector is dealt with as an independent country.

There are various reasons to deal the Chinese regions and provinces as individual 'countries'. One is their population size; the average size of the population in a province is 40 million. Another is their respective independence. In Shanghai, all of the taxis are Santana, and in Tianjin they are all Sharade ("Xiali" in Chinese). The vehicles themselves are made in Shanghai and Tianjin, respectively. Policy makers of each province want to be independent of the other

\footnotetext{
1 'Japan Sea' is called as 'East Sea' in the North and South Korea.

${ }^{2}$ A Japanese Research Institute established by Niigata Prefecture and for the research of the Japan Sea rim economic relations.
} 
provinces. In this sense, a Chinese regional or provincial model itself has a special value, ${ }^{3}$ and our model may be considered a step toward pioneering in this field.

\section{Framework of Our Model}

As a Japan Sea rim model, our model has some other characteristics.

First, the incorporated countries and regions include Japan, South Korea, Russia, and the Chinese Northeast region. We could not take reliable data on North Korea. For purposes to build "Japan Sea Rim model", dividing Russia into its mainland and the Far East would have been beneficial. However, we could not take such divided data. That issue is a subject to be addressed by the second version of this model.

Second, in order to analyze the trade relations of the Japan Sea rim economies, each macro model is a Keynesian-type demand side model.

Third, our model deals prices of trade goods in dollar terms in the trade bloc. Here, we must first determine export prices in dollar terms by export price equations, then determine import prices in dollar terms by using the following identities. That is,

$$
\mathrm{PM}_{j}=\sum_{i} \alpha i j \times \mathrm{PE}_{i}
$$

where PM and PE are the import prices and export prices, respectively, and $\alpha i j$ is the rate of import from economy $i$ of the total import of economy $j$, and $\sum_{i} \alpha i j=1$. Trade equations measured in each economy's currency in the macro bloc are introduced from these dollar term trade equations by using the exchange rates.

These are the main characteristics of this model, the details of which are shown in the Appendix of this paper.

Tables 1 and 2 show the results of the final test, conducted from 1991 to 1997 . The Japanese and Korean sectors' results are good, while the Russian sector's results are very bad, which may be explained by the reliability of the data and the shortage of its term; that is, the Russian data only extends from 1990 to 1998 . The trade bloc's results are not good between each country and Russia, or between the Chinese Northeast and Korea. The same explanation (i.e., insufficient data or insufficient study period), may hold here. For political reasons, trade relations between China and Korea are not yet well established. Given these considerations, our model has some problems, and will need to be improved after more reliable and longer-term data has been gathered. However, the fitness shown by this model is at a sufficient level to allow policy analyses.

\footnotetext{
${ }^{3}$ Although NAMIOS's regional macro model does not have trade bloc, it is a pioneer of the Chinese regional macro model. As a provincial model for the Chinese economy, one of us made a Xinjiang macro model (Ohnishi, 2000).
} 
Table 1 Absolute Mean Rate of Error of the Final Test (Macro Variables, \%, at 1990 constant price)

\begin{tabular}{|l|c|c|c|c|}
\hline & JAPAN & KOREA & $\begin{array}{c}\text { CHINESE } \\
\text { NORTHEAST }\end{array}$ & RUSSIA \\
\hline GDP & 2.3 & 3.7 & 4.7 & 18.6 \\
\hline CP(Private Consumption) & 1.2 & 2.5 & 3.8 & 8.9 \\
\hline IF(Private Investment) & 2.9 & 4.8 & 8.2 & 16.3 \\
\hline IM(Import) & 5.5 & 8.7 & 20.5 & 12.7 \\
\hline EX(Export) & 6.8 & 9.8 & 15.6 & 15.0 \\
\hline CU(Unit Cost) & 2.8 & 4.5 & -- & - \\
\hline UR(Unemployment Rate) & 12.6 & 15.7 & 20.3 & -- \\
\hline WI(Wage Index) & 3.2 & 8.3 & 10.2 & 35.7 \\
\hline WPI(Wholesale Price Index) & 2.6 & -- & 8.5 & - \\
\hline PDD & -- & 7.6 & -- & - \\
(Price Index of Domestic Demand) & & & & 40.5 \\
\hline PGDP(GDP Deflator) & 7.5 & 7.1 & 9.1 & \\
\hline
\end{tabular}

Table 2 Absolute Mean Rate of Error of the Final Test

(Trade Matrix, \%, dollar base at current price)

\begin{tabular}{|l|c|c|c|c|c|}
\hline importer & JAPAN & KOREA & $\begin{array}{c}\text { CHINESE } \\
\text { NORTHEAST }\end{array}$ & RUSSIA & $\begin{array}{c}\text { REST OF } \\
\text { THE WORLD }\end{array}$ \\
\hline JAPAN & -- & 10.6 & 12.4 & 63.3 & 8.0 \\
\hline KOREA & 6.3 & -- & 39.0 & 32.7 & 7.3 \\
\hline CHINESE NORTHEAST & 8.6 & 14.3 & -- & 58.0 & 5.9 \\
\hline RUSSIA & 53.0 & 36.6 & 27.1 & -- & 11.1 \\
\hline REST OF THE WORLD & 7.2 & 7.7 & 8.8 & 10.2 & - \\
\hline
\end{tabular}

\section{Policy Simulation}

Although our model is problematic as-is, its application yields useful information. Therefore, in this section, we simulate two kinds of policies and show the results of these simulation tests. One is the effect of Government Expenditure, and the other is the effect of the Japan-Korea Free Trade Area.

(1) International Effects of Government Expenditure

Tables 3 and 4 show the international effects in this area of an increase in each country's government expenditure. Here, the sizes of these increases are 1 percent of each country's GDP, 
and simulation tests are conducted to cover the projection period $1999-2005$.

According to the results, the effect of this respective increase in each country or region's GDP is 4.308-7.832 in Japan, $1.812-3.233$ in Korea, and $1.012-2.688$ in the Chinese Northeast (data not shown). Therefore, all of the Keynesian multipliers are larger than one, suggesting that the more advanced a country is, the more effective such a fiscal policy is.

Such effectiveness has an impact on the economy of the other countries or regions. In this experiment, the largest influence on other countries' GDP was made by the Japanese government expenditure, with the Korean, the Chinese Northeast's, and the Russian's government expenditure following (the effects of the last two are not shown in the tables). These results are in accord with the fact that Japan is the most influential country in this area.

However, such an influential order is a matter of course, given the size differences among these four economies. Therefore, the true influence of each country on the others must be compared by adjusting the size of the respective government expenditure; Table 5 includes adjustments made to the values shown in Table 3. In this table, the effects are multiplied by $1 / 15.5=0.0645$, which is the average ratio of the Korean GDP to the Japanese GDP through the simulation period. According to this table, Korea's government expenditure is more effective on the Russian economy than the Japanese government expenditure is. The reason may be that, compared to Japan, the Korean economy has stronger relations with the Russian economy, especially as an absorber. However, for the other economies, the scales of the Korean and the Japanese effects are almost same.

Table 3 Effects of 1 percent GDP increase in the Japanese Government Expenditure

(deviation from the standard projection, at 1990 constant price, percent)

\begin{tabular}{|l|c|c|}
\hline & in 2000 & in 2005 \\
\hline GDP of JAPAN & 4.308 & 7.832 \\
\hline GDP of KOREA & 2.889 & 7.321 \\
\hline GDP of the CHINESE NORTHEAST & 2.113 & 4.568 \\
\hline GDP of RUSSIA & 2.011 & 3.717 \\
\hline
\end{tabular}

Table 4 Effects of 1 percent GDP increase in the Korean Government Expenditure

(deviation from the standard projection, at 1990 constant price, percent)

\begin{tabular}{|l|c|c|}
\hline & in 2000 & in 2005 \\
\hline GDP of JAPAN & 0.211 & 0.776 \\
\hline GDP of KOREA & 1.812 & 3.223 \\
\hline
\end{tabular}




\begin{tabular}{|l|l|l|}
\hline GDP of the CHINESE NORTHEAST & 0.111 & 0.674 \\
\hline GDP of RUSSIA & 1.226 & 1.813 \\
\hline
\end{tabular}

Table 5 Effects of 0.0645 percent GDP increase in the Japanese Government Expenditure

(deviation from the standard projection, at 1990 constant price, percent)

\begin{tabular}{|l|c|c|}
\hline & in 2000 & in 2005 \\
\hline GDP of KOREA & 0.176 & 0.501 \\
\hline GDP of the CHINESE NORTHEAST & 0.129 & 0.313 \\
\hline GDP of RUSSIA & 0.123 & 0.255 \\
\hline
\end{tabular}

(2) Effects of the Japan-Korea Free Trade Area

Table 6 shows the effect of the planned Japan-Korea Free Trade Area. Here, reduction of custom duties are simulated by a cut in export prices; the ratios of this price-cut are assumed as 2.2 percent in the Japanese export price, and 3.7 percent in the Korean export price. Values after the price-cut represent the two countries' respective rates of custom duties for rest of the world during the year 1998. Therefore, this simulation can be understood to be predicated on a perfect free trade area.

According to the results, the amount of trade increases in both countries, and the impact on Korean trade activities is larger than that on Japanese trade activities in the term of percentage. Another noteworthy effect on the GDP is that Korea shows a loss in 2000, and a dramatic gain in 2005. The gain is the result of an increase in its gross domestic investment and consumption. Therefore, gains from this sort of free trade can be checked not only by trade surplus but also by domestic indicators.

Table 6 Effects of the Japan-Korea Free Trade Area

(deviation from the standard projection, percent, at constant price of each currency)

\begin{tabular}{|l|c|c|}
\hline & in 2000 & in 2005 \\
\hline GDP of JAPAN & 0.247 & 1.940 \\
\hline GDP of KOREA & -0.585 & 7.437 \\
\hline EXPORT of JAPAN & 2.787 & 5.700 \\
\hline EXPORT of KOREA & 3.628 & 7.208 \\
\hline IMPORT of JAPAN & 5.008 & 5.875 \\
\hline IMPORT of KOREA & 7.334 & 9.983 \\
\hline CONSUMPTION of JAPAN & 0.135 & 1.451 \\
\hline
\end{tabular}




\begin{tabular}{|l|l|l|}
\hline CONSUMPTION Of KOREA & -1.665 & 3.533 \\
\hline INVESTMENT of JAPAN & 0.285 & 2.236 \\
\hline INVESTMENT of KOREA & -2.135 & 7.264 \\
\hline
\end{tabular}

\section{References}

Ohnishi, H., 2000, Structure and Simulation of the Xinjiang Econometric Model, Research and Study, No.20, Kyoto University Economic Society (in Japanese).

Shishido,S. et al, 1999, A multiregional Econometric Model for Northeast Asia (NAMIOS I): Estimation and Policy Analysis, Journal of Econometric Study of Northeast Asia, Vol.1, No.1.

\section{APPENDIX Equation list of the Japan-sea Rim Econometric Model}

\section{MACRO MODEL}

1) FINAL DEMAND BLOC

( i )Gross domestic product

(1)GDP.J=CP.J+IF.J+CG.J+EX.J-IM.J+DISC.J

(2)GDP.K=CP.K+IF.K+CG.K+EX.K-IM.K+DISC.K

(3)GDP.CT=CP.CT+IF.CT+CG.CT+EX.CT-IM.CT+DISC.CT

(4)GDP.R=CP.R+IF.R+CG.R+EX.R-IM.R+DISC.R

(ii) Gross domestic product(nominal)

(5)GDPN.J=GDP.J*PGDP.J/100

(6)GDPN.K=GDP.K*PGDP.K/100

(7)GDPN.CT $=$ GDP.CT $*$ PGDP.CT $/ 100$

(8)GDPN.R=GDP.R*PGDP.R/100

(iii)Private Consumption

(9)CP.J $=7679.12+.375142 *(\mathrm{GDP} . J)+.334940 *(\mathrm{CP} . \mathrm{J}(-1))$

$$
\text { (5.65) (7.78) (3.99) }
$$

$$
\text { OLS } \quad(1971-1998) \quad \mathrm{R}^{\wedge} 2=.999 \quad \mathrm{SD}=1,880.09 \quad \mathrm{DW}=1.496
$$

(10)CP.K=1449.69+.214539*(GDP.K)+.633847*(CP.K(-1))-5103.32*(D97)-18592.5*(D98)
(3.31)
(6.82)
(9.65)
$(-5.45)$
$(-12.55)$

OLS $\quad(1972-1998) \quad \mathrm{R}^{\wedge} 2=1 . \quad \mathrm{SD}=789.7195 \quad \mathrm{DW}=1.331$

(11)CP.CT $=144.753+.295252 *($ GDP.CT $)+.276971 *(\mathrm{CP} . \mathrm{CT}(-1))$

$$
\text { (9.39) (10.26) (3.67) }
$$

$$
\text { OLS } \quad(1978-1998) \quad \mathrm{R}^{\wedge} 2=.998 \quad \mathrm{SD}=20.8918 \quad \mathrm{DW}=1.848
$$

(12)CP.R=109.711+.235059*(GDP.R)

$$
\text { (2.47) (2.49) }
$$

$$
\text { OLS } \quad(1990-1999) \quad \mathrm{R}^{\wedge} 2=.366 \quad \mathrm{SD}=28.8606 \quad \mathrm{DW}=1.377
$$


(iv)Private Investment

(13)IF.J $=-26628.6+.3644 *(G D P . J)-1858.33 *($ i.J-DOT(PCP.J))+8378.76*(D9091)-9489.08*(D98)

$$
(-7.05) \quad(35.63) \quad(-4.90)
$$

OLS $\quad(1975-1998) \quad \mathrm{R}^{\wedge} 2=.989 \quad \mathrm{SD}=3,086.22 \quad \mathrm{DW}=1.263$

(14)IF.K= - 13403.1+.4384*(GDP.K)-951.766*(I.K-DOT(PDD.K))-19068.3*(D98)-10488.8*(D97)

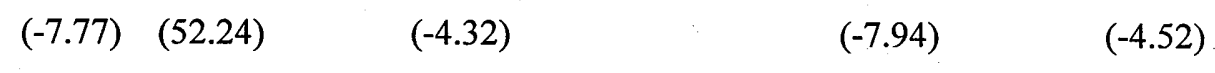

OLS $\quad(1981-1998) \quad \mathrm{R}^{\wedge} 2=.995 \quad \mathrm{SD}=2,004.56 \quad \mathrm{DW}=1.345$

(15)IF.CT $=-112.647+.317619 *($ GDP.CT) $-7.03461 *($ I.C-DOT(WPI.CT))

$$
(-4.05) \quad(29.37) \quad(-3.00)
$$

OLS $\quad(1980-1998) \quad \mathrm{R}^{\wedge} 2=.98 \quad \mathrm{SD}=50.3744 \quad \mathrm{DW}=1.437$

(16)IF.R $=-70.9618+.373799 *($ GDP.R)

$(-4.44) \quad(10.98)$

OLS $\quad(1990-1999) \quad \mathrm{R}^{\wedge} 2=.93 \quad \mathrm{SD}=10.3976 \quad \mathrm{DW}=2.008$

( $v$ )Export Goods and Services(nominal)

(17)EXN.J=694.966+1.087*(EXMN.J*RATEJ/1000)

(1.25) (71.01)

Orrcut $(1971-1998) \quad \mathrm{R}^{\wedge} 2=.999 \quad \mathrm{SD}=490.3399 \quad \mathrm{DW}=1.68 \quad \mathrm{RO}=.484$

(18)EXN.K=154.831+1.19595*(EXMN.K*RATEK/1000)

(.18) (80.19)

OLS $\quad(1971-1998) \quad \mathrm{R}^{\wedge} 2=.996 \quad \mathrm{SD}=3,488.24 \quad \mathrm{DW}=2.114$

(19)EXN.CT=1.2*(EXMN.CT*RATEC/100)

(20)EXN.R=18282.3+1.09935*(EXMN.R*RATER)

(2.96) (133.77)

OLS $\quad(1992-1999) \quad \mathrm{R}^{\wedge} 2=1 . \quad \mathrm{SD}=12,748.4 \quad \mathrm{DW}=1.294$

(vi)Deflator of Export Goods and Services

(21)PEX.J=1.30209+.718112*(WPI.J)+.164411*(RATEJ)

$$
\text { (.16) (7.92) (11.07) }
$$

Orrcut $(1972-1998) \quad \mathrm{R}^{\wedge} 2=.979 \quad \mathrm{SD}=2.11151 \quad \mathrm{DW}=1.45 \quad \mathrm{RO}=.587$

$(22) \operatorname{LOG}($ PEX.K $)=2.48588+.227159 *($ LOG(PDD.K $))+.161568 *($ LOG(RATEK) $)$

$$
\text { (6.97) (3.58) (2.24) }
$$

OLS $\quad(1986-1998) \quad \mathrm{R}^{\wedge} 2=.808 \quad \mathrm{SD}=.041163 \quad \mathrm{DW}=2.164$

(23)PEX.CT $=$ PUEC*RATEC $/ 4.7832$

(vii)Export Goods and Services

(24)EX.J=EXN.J/PEX.J*100

(25)EX.K=EXN.K/PEX*100

(26)EX.CT=EXN.CT/PEX.CT*100 
(27)EX.R=EXN.R/PGDP.R*100

(viii)Import Goods and Services(nominal)

(28)IMN.J=3134.09+1.13587*(IMMN.J*RATEJ/1000)

(1.22) (26.85)

Orrcut (1972-1998) $\quad \mathrm{R}^{\wedge} 2=.994 \quad \mathrm{SD}=823.1515 \quad \mathrm{DW}=2.073 \quad \mathrm{RO}=0.917$

(29)IMN.K $=-1108.72+1.18366 *(I M M N . K * R A T E K / 1000)$

$(-1.26) \quad$ (76.46)

OLS $\quad(1971-1998) \quad R^{\wedge} 2=.995 \quad \mathrm{SD}=3,344.58 \quad \mathrm{DW}=1.638$

(30)IMN.CT=1.2*(IMMN.CT*RATEC/100)

(31)IMN.R $=5622.01+1.20855 *($ IMMN.R*RATER)

(.27) (27.48)

OLS $\quad(1992-1999) \quad \mathrm{R}^{\wedge} 2=.991 \quad \mathrm{SD}=38,726.8 \quad \mathrm{DW}=2.383$

(ix)Deflator of Import Goods and Services

$(32)$ LOG $($ PIM.J $)=-3.43211+.844441 *$ LOG(PUMJ)+.837410*LOG(RATEJ)

$$
(-15.21) \quad(21.35) \quad(24.82)
$$

Orrcut (1971-1998) $\quad \mathrm{R}^{\wedge} 2=.995 \quad \mathrm{SD}=.020425 \quad \mathrm{DW}=1.662 \quad \mathrm{RO}=$

(33)LOG(PIM.K) $=-5.38561+.888632 *$ LOG(PUMJ) $+.894755 *$ LOG(RATEK)

$$
(-12.63) \quad(12.04) \quad(9.26)
$$

OLS $\quad(1971-1998) \quad \mathrm{R}^{\wedge} 2=.974 \quad \mathrm{SD}=.102000 \quad \mathrm{DW}=1.215$

(34)PIM.CT=PUMC*RATEC/4.7832

( $\mathrm{x}$ )Import Goods and Services

(35)IM.J=IMN.J/PIM.J*100

(36)IM.K:=IMN.K/PIM.K*100

(37)IM.CT=IMN.CT/PIM.CT*100

(38)IM.R=IMN.R/PGDP.R*100

2) CAPACITY UTILIZATION AND LABOR MARKET BLOC

( i )Capacity Utilization

(39)CU.J=52.9324+2.36686*(GDP.J/N.J)-.689953*(K.J(-1)/N.J)

$$
\text { (3.84) (4.50) (-4.77) }
$$

Orrcut $(1972-1998) \quad \mathrm{R}^{\wedge} 2=.625 \quad \mathrm{SD}=3.98983 \quad \mathrm{DW}=1.726 \quad \mathrm{RO}=0.307$

(40)CU.K=62.6443+.733475*(GDP.K/N.K)-.235765*(K.K(-1)/N.K)

$$
\begin{array}{cccc} 
& (28.98) & (13.80) & (-11.60) \\
\text { OLS } \quad(1980-1998) & \mathrm{R}^{\wedge} 2=.923 & \mathrm{SD}=1.87468 & \mathrm{DW}=1.205
\end{array}
$$

(ii )Capital stock

(41)K.J $=(1-D . J) * K . J(-1)+I F . J$

(42)K.K $=(1-D \cdot K) * K \cdot K(-1)+I F \cdot K$ 
(iii)Labor Demand

(43)N.J=4311.36+.005817*(GDP.J)-539.451*(WI.J/PCP.J)

$$
\text { (55.04) (27.27) (-3.37) }
$$

OLS $\quad(1970-1998) \quad \mathrm{R}^{\wedge} 2=.996 \quad \mathrm{SD}=33.2950 \quad \mathrm{DW}=1.022$

(44)LOG(N.K) $=3.52131+.327929 *$ LOG(GDP.K)

(24.80) (27.35)

Orrcut (1972-1998) $\quad \mathrm{R}^{\wedge} 2=.995 \quad \mathrm{SD}=.012759 \quad \mathrm{DW}=1.63 \quad \mathrm{RO}=.627$

(45)LOG(L.CT) $=5.564+.606 *$ LOG(GDP.CT) $-.610 *$ LOG(W.CT/WPI.CT $)+.084 *$ D86-.070*(D9798)

$$
\text { (32.81) (6.30) (-3.00) }
$$

OLS $\quad(1979-1998) \quad \mathrm{R}^{\wedge} 2=.957 \quad \mathrm{SD}=.031001 \quad \mathrm{DW}=1.246$

(iv) Unemployment Rate

(46)UR.J $=-2.18515+4.90374 *$ (WI.J/PCP.J)+.855157*(D98)

$$
(-1.00) \quad(2.23)
$$

Orrcut (1971-1998) $\quad \mathrm{R}^{\wedge} 2=.917 \quad \mathrm{SD}=.194692 \quad \mathrm{DW}=1.099 \quad \mathrm{RO}=.804$

(47)DOT(UR.K)=8.19753-.793209*(DOT(IF.K))+141.851*(D98)

$$
\text { (2.33) (-3.57) (10.31) }
$$

OLS(1973-1998) $\quad \mathrm{R}^{\wedge} 2=.895 \quad \mathrm{SD}=11.5514 \quad \mathrm{DW}=1.802$

(48)UR.CT $=.433740+.097011 *$ (W.CT/WPI.CT)

$$
\text { (.74) (3.84) }
$$

OLS $\quad(1984-1998) \quad \mathrm{R}^{\wedge} 2=.496 \quad \mathrm{SD}=.422410 \quad \mathrm{DW}=1.386$

\section{3 ) WAGE-PRICE BLOC}

(i) Wage

(49)DOT(WI.J) $=6.75563+1.07158 *($ DOT(PCP.J))-2.03900*(UR.J)

$$
\text { (3.35) (9.98) } \quad(-2.80)
$$

OLS $\quad(1970-1998) \quad \mathrm{R}^{\wedge} 2=.886 \quad \mathrm{SD}=2.12206 \quad \mathrm{DW}=1.226$

(50)DOT(WI.K)=18.5674+.425441*(DOT(PDD.K))-3.73624*(UR.K)

$$
\text { (3.63) (2.32) (-3.05) }
$$

Orrcut (1976-1998) $\quad \mathrm{R}^{\wedge} 2=.831 \quad \mathrm{SD}=3.83381 \quad \mathrm{DW}=1.973 \quad \mathrm{RO}=.786$

$(51)$ DOT(W.CT) $=26.9818+.528826 *($ DOT(WPI.CT(-1)))-7.11847*(UR.CT)

$$
\text { (4.23) (2.38) (-3.04) }
$$

OLS $\quad(1981-1998) \quad \mathrm{R}^{\wedge} 2=.379 \quad \mathrm{SD}=5.89120 \quad \mathrm{DW}=1.376$

(52)WI.R=788.493+.019596*(PGDP.R)

(.78) (10.34)

OLS $\quad(1990-1999) \quad \mathrm{R}^{\wedge} 2=.922 \quad \mathrm{SD}=2,312.44 \quad \mathrm{DW}=1.520$

(ii) Prices

(53)WPI.J=7.64415+31.0639*(WI.J*N.J/GDP.J)+.309206*(PIM.J)+.134876*(CU.J) 

(.79) (9.17)
(16.27)
(2.00)

Orrcut $(1971-1998) \quad \mathrm{R}^{\wedge} 2=.987 \quad \mathrm{SD}=1.66441 \quad \mathrm{DW}=1.599 \quad \mathrm{RO}=0.507$

(54)PDD.K $=-43.4203+59.8757 *($ WI.K*N.K/GDP.K $)+.276865 *($ CU.K $)+.570675 *$ (PIM.K)

$$
(-4.95) \quad(33.13)
$$

(13.93)

OLS $\quad(1980-1998) \quad \mathrm{R}^{\wedge} 2=.997 \quad \mathrm{SD}=1.82878 \quad \mathrm{DW}=1.750$

(55)WPI.CT $=-26.7470+.031450 *($ W.CT*L.CT/GDP.CT $)+15.5089 *(\mathrm{D} 9798)$

$$
(-8.94) \quad(40.92)
$$

OLS $\quad(1978-1998) \quad \mathrm{R}^{\wedge} 2=.992 \quad \mathrm{SD}=4.34634 \quad \mathrm{DW}=1.295$

(56)PGDP.R $=-11727.4+47.4799 *($ WI.R)

$$
(-.23) \quad(10.34)
$$

OLS $\quad(1990-1999) \quad \mathrm{R}^{\wedge} 2=.922 \quad \mathrm{SD}=113,827.1 \quad \mathrm{DW}=1.622$

(57)PCP.J=41.2591+.115548*(WPI.J)+.487560*(WI.J)

$$
\text { (4.56) (3.63) (7.10) }
$$

Orrcut $(1973-1998) . \quad \mathrm{R}^{\wedge} 2=.998 \quad \mathrm{SD}=.727449 \quad \mathrm{DW}=1.319 \quad \mathrm{RO}=.843$

(58)LOG(PGDP.J) $=3.34475+.291324 *$ LOG(WPI.J)

(13.58) (5.59)

Orrcut $(1971-1998) \quad \mathrm{R}^{\wedge} 2=.972 \quad \mathrm{SD}=.016438 \quad \mathrm{DW}=1.387 \quad \mathrm{RO}=0.887$

(59)LOG(PGDP.K) $=-. .124842+1.02566 *$ LOG(PDD.K)

$$
(-1.36) \quad(49.76)
$$

Orrcut (1972-1998) $\quad \mathrm{R}^{\wedge} 2=.999 \quad \mathrm{SD}=.020564 \quad \mathrm{DW}=1.283 \quad \mathrm{RO}=0.680$

(60)PGDP.CT $=12.9876+.926304 *$ WPI.CT

(3.31) (28.66)

Orrcut (1978-1998) $\quad \mathrm{R}^{\wedge} 2=.996 \quad \mathrm{SD}=3.132 \quad \mathrm{DW}=1.212 \quad \mathrm{RO}=0.588$

4) FINANCIAL MARKET BLOC

(61)I.J=2.05361+.446752*(DR.J)-.249642*(M.J/PGDP.J)

$$
\text { (2.67) (5.56) (-1.91) }
$$

OLS $\quad(1970-1998) \quad \mathrm{R}^{\wedge} 2=.811 \quad \mathrm{SD}=.650582 \quad \mathrm{DW}=1.469$

$(62) \mathrm{LOG}(\mathrm{I} . \mathrm{K})=-7.3328+1.72388 *(\mathrm{LOG}(\mathrm{GDP} . \mathrm{K}))-1.71018 *(\mathrm{LOG}(\mathrm{M} . \mathrm{K} / \mathrm{PGDP} . \mathrm{K}))$

$$
\begin{array}{cccc} 
& (-1.09) & (1.77) & (-2.16) \\
+.732225 *(\mathrm{D} 98) & & \\
(2.65) & & & \\
\text { Orrcut }(1972-1998) & \mathrm{R}^{\wedge} 2=.683 & \mathrm{SD}=.162925 & \mathrm{DW}=1.695 \quad \mathrm{RO}=0.662
\end{array}
$$

\section{TRADE MODEL}

1) EXPORT PRICE

(63)LOG(PUEJ)=4.78405+.822740*(LOG(WPI.J))-.788822*(LOG(RATEJ))
(11.70)
(7.22)
$(-13.45)$ 
Orrcut $(1971-1998) \quad \mathrm{R}^{\wedge} 2=.994 \quad \mathrm{SD}=.026378 \quad \mathrm{DW}=1.717 \quad \mathrm{RO}=0.834$

(64)LOG(PUEK)=4.37633+.500939*(LOG(PDD.K))-.321087*(LOG(RATEK))-.384644*(D9798)

(6.49) (11.29) (-2.49) (-5.26)

OLS $\quad(1971-1998) \quad \mathrm{R}^{\wedge} 2=.942 \quad \mathrm{SD}=.081484 \quad \mathrm{DW}=1.277$

$(65)$ LOG(PUEC) $=1.71601+.708767 *($ LOG(WPI.CT) $)-.272416 *($ LOG(RATEC $))$

(5.36) (7.68) (-3.74)

OLS $\quad(1980-1998) \quad \mathrm{R}^{\wedge} 2=.933 \quad \mathrm{SD}=.047889 \quad \mathrm{DW}=1.674$

2 ) EXPORT of Goods(nominal price, dollar base)

(i) Export From Japan

(66)EXMN.J=EXMNK.J+EXMNCT.J+EXMNR.J+EXROW.J

(67)EXMNK.J=EXK.J\#*PUEJ/100

(68)LOG(EXK.J\#)=6.40610+.668363*(LOG(GDP.K))-.731046*(LOG(PUEJ/(PGDP.K/RATEK)))

(2.94) (9.18) (-3.40)

OLS $\quad(1972-1998) \quad \mathrm{R}^{\wedge} 2=.928 \quad \mathrm{SD}=.147696 \quad \mathrm{DW}=1.345$

(69)EXMNCT.J=EXCT.J\#*PUEJ/100

(70)LOG(EXCT.J\#) $=-8.90730+1.93583 *($ LOG(GDP.CT $))$

$(-6.15) \quad(10.68)$

OLS $\quad(1988-1998) \quad \mathrm{R}^{\wedge} 2=.919 \quad \mathrm{SD}=.162518 \quad \mathrm{DW}=1.256$

(71)EXMNR.J=EXMNR.J\#/RATER

(72)EXMNR.J\#=628.1852+.002681*GDPN.R

(.82) (6.03)

OLS $\quad(1992-1998) \quad \mathrm{R}^{\wedge} 2=.8550 \quad \mathrm{SD}=1213.731 \quad \mathrm{DW}=1.932$

(73)EXROW.J=114154.8+.251174*(WTM)

(6.54) (12.13)

Orrcut (1984-1998) $\quad \mathrm{R}^{\wedge} 2=.979 \quad \mathrm{SD}=12,354.5 \quad \mathrm{DW}=1.606 \quad \mathrm{RO}=.431$

(ii) Export From Korea

(74)EXMN.K=EXMNJ.K+EXMNCT.K+EXMNR.K+EXROW.K

(75)EXMNJ.K=EXJ.K\#*PUEK/100

(76)LOG(EXJ.K\#) $=-11.01+1.882 *$ LOG(GDP.J)-.83 $2 *$ LOG(PUEK/(WPI.J/RATEJ))+.32*D89

$$
(-2.74)(8.64)
$$

OLS $\quad(1973-1998) \quad \mathrm{R}^{\wedge} 2=.966 \quad \mathrm{SD}=.123455 \quad \mathrm{DW}=1.405$

(77)EXMNCT.K=EXCT.K\#*PUEK $/ 100$

(78)LOG(EXCT.K\#) $=-28.9998+4.29759 *($ LOG(GDP.CT $))$

$$
(-6.58) \quad(7.96)
$$

OLS $\quad(1992-1998) \quad \mathrm{R}^{\wedge} 2=.912 \quad \mathrm{SD}=.268066 \quad \mathrm{DW}=1.487$

(79)EXMNR.K=EXMNR.K\#/RATER 
(80)EXMNR.K\#= - 178.8991+.004278*GDPN.R

$(-.83) \quad(34.18)$

OLS $\quad(1992-1998) \quad \mathrm{R}^{\wedge} 2=.9949 \quad \mathrm{SD}=341.776 \quad \mathrm{DW}=2.078$

(81)EXROW.K= - 14507.7+.109224*(WTM)+19299.9*(D98)

$(-2.97) \quad(18.04) \quad(5.78)$

Orrcut (1980-1998) $\quad \mathrm{R}^{\wedge} 2=.992 \quad \mathrm{SD}=3,246.17 \quad \mathrm{DW}=1.762 \quad \mathrm{RO}=.620$

(iii) Export From the Chinese Northeast

(82)EXMN.CT=EXMNJ.CT+EXMNK.CT+EXMNR.CT+EXROW.CT

(83)EXMNJ.CT=EXJ.CT\#*PUEC/100

(84)LOG(EXJ.CT\#)=-19.6500+2.25423*(LOG(GDP.J))-.351237*(LOG(PUEC/(WPI.J/RATEJ)))

$$
(-7.24) \quad(9.71) \quad(-2.91)
$$

OLS $\quad(1988-1998) \quad \mathrm{R}^{\wedge} 2=.913 \quad \mathrm{SD}=.042075 \quad \mathrm{DW}=2.046$

(85)EXMNK.CT=EXK.CT\#*PUEC $/ 100$

(86)LOG(EXK.CT\#)=-16.835+2.41354*(LOG(GDP.K))-.984166*(LOG(PUEC/(PDD.K/RATEK)))

$$
(-3.73) \quad(5.80) \quad(-2.95)
$$

OLS $\quad(1992-1998) \quad \mathrm{R}^{\wedge} 2=.841 \quad \mathrm{SD}=.114718 \quad \mathrm{DW}=1.553$

(87)EXMNR.CT=EXMNCT\#/RATER

(88)EXMNR.CT\#=690.7547+.002521*GDPN.R

(.74) (4.66)

OLS $\quad(1992-1998) \quad \mathrm{R}^{\wedge} 2=.7756 \quad \mathrm{SD}=1476.703 \quad \mathrm{DW}=1.759$

(89)EXROW.CT $=1294.63+.003892 *(W T M)$

(4.01) (10.04)

OLS $\quad(1986-1998) \quad \mathrm{R}^{\wedge} 2=.893 \quad \mathrm{SD}=363.4577 \quad \mathrm{DW}=1.317$

(iv) Export From Russia

(90)EXMN.R=EXMNJ.R+EXMNK $\cdot R+E X M N C T \cdot R+E X R O W \cdot R$

(91)EXMNJ.R=EXMNJ.R\#/RATEJ

(92)EXMNJ.R\#=-1469956+3.56911*(GDPN.J)

$$
(-4.52) \quad(5.37)
$$

OLS $\quad(1992-1998) \quad \mathrm{R}^{\wedge} 2=.822 \quad \mathrm{SD}=23,771.1 \quad \mathrm{DW}=2.038$

(93)EXMNK.R=EXMNK.R\#/RATEK

(94)EXMNK.R\#=-502594.7+2.71749*(GDPN.K)

$$
(-3.69) \quad(7.41)
$$

OLS $\quad(1992-1998) \quad \mathrm{R}^{\wedge} 2=.9 \quad \mathrm{SD}=74,741.2 \quad \mathrm{DW}=3.538$

(95)EXMNCT.R=EXMNCT.R\#/RATEC

(96)EXMNCT.R\#=2668.11+.672889*(GDPN.CT)

$$
\text { (1.38) (1.91) }
$$


OLS $\quad(1990-1998) \quad \mathrm{R}^{\wedge} 2=.2485 \quad \mathrm{SD}=2282.844 \quad \mathrm{DW}=.816$

(97)EXROW.R $=-51730.4+.117007 *(\mathrm{WTM})$

$$
(-5.14) \quad(11.78)
$$

OLS $\quad(1992-1998) \quad \mathrm{R}^{\wedge} 2=.958 \quad \mathrm{SD}=3,853.79 \quad \mathrm{DW}=2.663$

( v ) Export From Rest of the World

(98)EXMN.ROW=EXJ.ROW+EXK.ROW+EXCT.ROW+EXR.ROW

(99)EXJ.ROW=EXJ.ROW\#*PUE.ROW/100

(100)LOG(EXJ.ROW\#)= - 32.848+3.70*(LOG(GDP.J)) $-.618 *($ LOG(PUE.ROW/(WPI.J/RATEJ)) $)$

$$
(-5.01)(6.94)
$$

OLS $\quad(1991-1998) \quad \mathrm{R}^{\wedge} 2=.868 \quad \mathrm{SD}=.047983 \quad \mathrm{DW}=1.616$

(101)EXK.ROW=EXK.ROW\#*PUE.ROW/100

(102)LOG(EXK.ROW\#)=-2.62+1.59*(LOG(GDP.K))

$$
(-.96) \quad(8.18)
$$

$-.894 *($ LOG(PUE.ROW/(PDD.K/RATEK)))

$(-5.66)$

Orrcut (1989-1998) $\quad \mathrm{R}^{\wedge} 2=.981 \quad \mathrm{SD}=.042088 \quad \mathrm{DW}=1.303 \quad \mathrm{RO}=0.503$

(103)EXCT.ROW=EXCT.ROW\#*PUE.ROW/100

(104)LOG(EXCT.ROW\#)=-9.76750+2.15517*(LOG(GDP.CT))

$$
(-5.57) \quad(9.82)
$$

OLS $\quad(1988-1998) \quad \mathrm{R}^{\wedge} 2=.905 \quad \mathrm{SD}=.196715 \quad \mathrm{DW}=1.526$

3) World Trade

(105)WTM=IMMN.J+IMMN.K+IMMN.CT+IMMN.R+IMMN.ROW

4) Convert From Exports Goods to Imports Goods

(106)IMMN.J=EXMNJ.K+EXMNJ.CT+EXMNJ.R+EXJ.ROW

(107)IMMN.K=EXMNK.J+EXMNK.CT+EXMNK.R+EXK.ROW

(108)IMMN.CT=EXMNCT.J+EXMNCT.K+EXMNCT.R+EXCT.ROW

(109)IMMN.R=EXMNR.J+EXMNR.K+EXMNR.CT+EXR.ROW

(110)IMMN.ROW=EXROW.J+EXROW.K+EXROW.CT+EXROW.R

5 ) Convert From Export Prices to Import Prices

(111)PUMJ=EXMNJ.K/IMMN.J*PUEK+EXMNJ.CT/IMMN.J*PUEC+EXJ.ROW/IMMN.J*PUE. ROW

(112)PUMK=EXMNK.J/IMMN.K*PUEJ+EXMNK.CT/IMMN.K*PUEC+EXK.ROW/IMMN.K*P UE.ROW

(113)PUMC=EXMNCT.J/IMMN.CT*PUEJ+EXMNCT.K/IMMN.CT*PUEK+EXCT.ROW/IMMN. CT*PUE.ROW 


\section{List of Variables}

\begin{tabular}{|c|c|c|}
\hline CG & Government Consumption(1990 price, each currency) & see footnote \\
\hline $\mathrm{CP}$ & Private Consumption(1990 price, each currency) & see footnote \\
\hline $\mathrm{CU}$ & Capacity Utilization & percent \\
\hline D & Depreciation Rate & percent \\
\hline DISC & $\begin{array}{l}\text { Statistical Discrepancy + Stock Inventory }(1990 \text { price } \\
\text { currency) }\end{array}$ & each see footnote \\
\hline DR & Discount Rate & percent \\
\hline EX & Export of Goods and Services(1990 price, each currency) & see footnote \\
\hline EX--\# & Export of Goods and Services(1990 price, dollar base) & million dollar \\
\hline EXMN & Export of Goods (nominal price, dollar base) & million dollar \\
\hline EXMN-—\# & Export of Goods (1990 price, dollar base) & million dollar \\
\hline EXN & Export of Goods and Services (nominal, each currency) & see footnote \\
\hline GDP & Gross Domestic Product (1990 price, each currency) & see footnote \\
\hline GDPN & Gross Domestic Product (nominal price, each currency) & see footnote \\
\hline I & Interest Rate & percent \\
\hline IF & Gross Domestic Investment & see footnote \\
\hline IM & Import of Goods and Services (1990 price, each currency) & see footnote \\
\hline IMMN & Import of Goods (nominal price, dollar base) & million dollar \\
\hline IMN & Import of Goods and Services (nominal price, each currency) & see footnote \\
\hline K & Capital Stock (1990 price, each currency) & see footnote \\
\hline M & Money Supply & $\begin{array}{l}\text { trillion yen, billion } \\
\text { won }\end{array}$ \\
\hline N & Number of Workers & ten thousand person \\
\hline PCP & Consumers Price Index & $1990=1$ \\
\hline PDD & Domestic Demand Deflator & $1990=1$ \\
\hline PEX & Deflator of Export of Goods and Services & $1990=1$ \\
\hline PGDP & GDP Deflator & $1990=1$ \\
\hline PIM & Deflator of Import of Goods and Services & $1990=1$ \\
\hline PUE & Unit Price of Export Goods & $1990=1$ \\
\hline PUM & Unit Price of Import Goods & $1990=1$ \\
\hline RATE & Exchange Rate & each currency/dollar \\
\hline UR & Unemployment Rate & percent \\
\hline W & Average Wage & yuan \\
\hline WI & Wage Index & $1990=1$ \\
\hline
\end{tabular}


WPI Wholesale Price Index

WTM World Trade

million dollar

J,K,C,CT,R,ROW indicate Japan, Korea, China, the Chinese Northeast, Russia and rest of the World respectively.

Some variables of EX, EX--\#, EXMN, EXMN--\# indicate their trade directions. For example, EXK.J indicates export of goods from Japan to Korea.

Each currency's unit of the macro and trade variables is billion yen, billion won, 100 million yuan and million ruble. 